hep-ph/0404284v2

\title{
The Kinetic Equation for Electroweak Baryogenesis
}

\author{
Jitesh Bhatt* and Raghavan Rangarajan ${ }^{\dagger}$ \\ Theoretical Physics Division, Physical Research Laboratory \\ Navrangpura, Ahmedabad 380 009, India
}

(Dated: November 2, 2018)

\begin{abstract}
We derive the kinetic equation for fermions and antifermions interacting with a planar Higgs bubble wall during the electroweak phase transition using the 'evenisation' procedure. Equations of motion in a relativistic quantum theory do not mirror classical relations unless one uses evenised operators. We give a brief introduction to evenisation and then use the evenised Heisenberg equations of motion to obtain the velocity and force for the particles in the presence of the Higgs bubble wall. Keeping quantum contributions to $O(\hbar)$ in the equations of motion we obtain the semi-classical force obtained earlier by other techniques.

\footnotetext{
* jeet@prl.ernet.in

$\dagger$ raghavan@prl.ernet.in
}

PACS numbers: 98.80.Cq, 03.65.Sq
\end{abstract}




\section{INTRODUCTION}

Electroweak baryogenesis allows the possibility that the observed matter-antimatter asymmetry of the universe may have been obtained during the electroweak phase transition at temperatures of around $100 \mathrm{GeV}$, corresponding to an energy scale that is experimentally accessible. Hence there has been considerable interest in ascertaining the details of electroweak baryogenesis models, in particular, the kinetic equation or Boltzmann equation for the transport of particles through the Higgs bubble wall in a first order electroweak phase transition.

It is well known that the characteristics of the Boltzmann equation, in the absence of collisions, are the single particle trajectory equations, i.e. if $f(\mathbf{p}, \mathbf{x}, t)$ is a distribution function for a system of particles then $\frac{d f(\mathbf{p}, \mathbf{x}, t)}{d t}=0$, where $\mathbf{x}$ and $\mathbf{p}$ satisfy single particle equations of motion. Therefore expanding the total derivative using the chain rule as

$$
\partial_{t} f+\frac{d \mathbf{x}}{d t} \partial_{\mathbf{x}} f+\frac{d \mathbf{p}}{d t} \partial_{\mathbf{p}} f=0
$$

the quantities $\frac{d \mathbf{x}}{d t}$ and $\frac{d \mathbf{p}}{d t}$ can be obtained for the system from the single particle equations of motion (Hamilton's equations). However, in relativistic quantum theories it is non-trivial to obtain the single particle equations of motion from the Hamiltonian because of interference between particle and antiparticle states causing Zitterbewegung. Obtaining equations of motion that can have a consistent single particle interpretation requires the use of 'evenised' operators [1], as we discuss below. We apply the procedure of evenisation to calculate particle trajectories to $O(\hbar)$ in the presence of the Higgs bubble wall during the electroweak phase transition (presumed to be first order). We then use this to obtain the kinetic equation.

In Ref. 2] the energy relation, $\omega_{s \pm}(\mathbf{p}, \mathbf{x})$, and a kinetic equation in the absence of collisions for particles and antiparticles interacting with the Higgs bubble wall are obtained from the equation of motion for the Wigner function $G^{<}(x, k)$, which is the Wigner transform of the Wightman function $i\left\langle\bar{\psi}\left(x^{\prime}\right) \psi\left(x^{\prime \prime}\right)\right\rangle$. (Also see Ref. [3].) In this approach, particle and antiparticle distribution functions are extracted from the Wigner function using a spectral decomposition that separates positive and negative energy states. The particle and antiparticle distribution functions are then found to satisfy a collisionless quantum corrected kinetic equation in the presence of the external force field provided by the Higgs wall.

The kinetic equation has also been derived in Ref. [4]. The energy relation was obtained using an WKB ansatz for components of the (spinor) wave function. The single particle 
trajectory was obtained by treating the energy relation as the Hamiltonian. Earlier analyses of the kinetic equation using the WKB ansatz were developed in Refs. [5, 6].

In the above approaches the dominant contribution to the $\mathrm{CP}$ violating source that leads to a baryon asymmetry is associated with the force term in the kinetic equation. Alternate formalisms for the derivation of the kinetic equation have been provided in Refs. [7, 8, 9, 10, 11]. In Refs. 7] and [8] a source term was included in the diffusion equation to describe the $\mathrm{CP}$ violating interaction between the fermions/antifermions and the bubble wall in the presence of the thermal plasma. In the former, the source was calculated by including reflection and transmission of particles and antiparticles by each layer of the wall. In Ref. 8] the expectation value of the $\mathrm{CP}$ violating current was calculated using the Closed Time Path formalism and its divergence was included as a source. In Refs. 9, 10] the Closed Time Path formalism was used to derive the kinetic equation itself from the equation of motion of the relevant Green's function.

The kinetic equation obtained below by us agrees with Ref. [3]. In particular we obtain the semi-classical force term, first discussed in Ref. [12]. Our approach provides an alternate and intuitive derivation of the (collisionless) kinetic equation relevant to electroweak baryogenesis to $O(\hbar)$. Furthermore, we are not aware of earlier attempts to use evenisation to study particle trajectories at higher than $O\left(\hbar^{0}\right)$.

\section{II.}

A first order electroweak phase transition proceeds via the formation of Higgs bubbles. As the bubbles expand they move through the ambient sea of quarks, leptons and other particles. To calculate the baryon asymmetry created as the ambient particles interact with the expanding Higgs bubble it is necessary to study the kinetic equation describing the passage of the particles through the bubble wall. Below we first obtain an expression for the single particle energy to $O(\hbar)$ in terms of the evenised position and momentum. We then obtain expressions for the evenised velocity and force and substitute these in Eq. (II) to obtain the kinetic equation.

We first give a brief introduction to evenisation. In relativistic quantum mechanics the eigenvalues of many operators do not agree with their classical values. For example, for a free particle the eigenvalues of the velocity operator, $d \hat{\mathbf{x}} / d t=(i \hbar)^{-1}[\hat{\mathbf{x}}, \hat{H}]=c \hat{\alpha}$, are $\pm c$ 
even for a massive particle. In addition, one can not obtain relations analogous to classical equations for expectation values of quantum operators (i.e. Ehrenfest's theorem), as also evidenced by the expression for the velocity operator (quantum mechanically $d\langle\hat{\mathbf{x}}\rangle / d t=c\langle\hat{\alpha}\rangle$ while classically, $d \mathbf{x} / d t=\mathbf{p} / E)$. All this is because of interference between particle and antiparticle states leading to Zitterbewegung [1]. One may resolve this by considering 'even' parts of operators, and by replacing operators by their 'even' counterparts in equations of motion. This then allows a consistent single particle interpretation of the Dirac equation.

As an illustration, the evenised velocity operator is given by $[d \hat{\mathbf{x}} / d t]=[\hat{\alpha}]=$ $\hat{\mathbf{p}} \hat{\Lambda} / \sqrt{\hat{\mathbf{p}}^{2}+m^{2}}$, where $\hat{\Lambda}$ is a sign operator with eigenvalues \pm 1 (and we now set $c=1$ ). This mimics the classical relation. Analogously, we shall obtain an expression for the Hamiltonian (actually its square) in terms of evenised position and momentum operators and use that to obtain an expression for the energy of the particle. We will further obtain expressions for the evenised velocity and force on the particle in the presence of the bubble wall and use these to obtain the kinetic equation.

Any operator $\hat{A}$ can be split into an even part $[\hat{A}]$ and into an odd part $\{\hat{A}\}$. Even and odd parts of the operators are defined by using the sign operator [1],

$$
\hat{\Lambda}=\frac{\hat{H}}{\sqrt{\hat{H}^{2}}}
$$

where $\hat{H}$ is the Hamiltonian. The eigenvalues of the sign operator are \pm 1 , corresponding to particle and antiparticle states. Then

$$
\begin{gathered}
{[\hat{A}]=\frac{1}{2}(\hat{A}+\hat{\Lambda} \hat{A} \hat{\Lambda})} \\
\{\hat{A}\}=\frac{1}{2}(\hat{A}-\hat{\Lambda} \hat{A} \hat{\Lambda})=\frac{1}{2}[\hat{A}, \hat{\Lambda}] \hat{\Lambda}
\end{gathered}
$$

The even part of the product of two operators $\hat{A}$ and $\hat{B}$ can be written as

$$
[\hat{A} \hat{B}]=[\hat{A}][\hat{B}]+\{\hat{A}\}\{\hat{B}\}
$$

We now apply the above to the system under consideration.

The Lagrangian describing the interaction of particles with the bubble wall can be modeled by

$$
\mathcal{L}=i \bar{\psi} \not \partial-m_{R} \bar{\psi} \psi-i m_{I} \bar{\psi} \gamma_{5} \psi
$$


where $m_{R}$ and $m_{I}$ are real numbers. ${ }^{1}$ The Higgs bubble will be treated as a background field which provides a complex spatially varying mass for the particles in the bubble wall frame, in the limit of large bubbles when the walls can be treated as planar. The corresponding Dirac equation

$$
\left(i \not \partial-m_{R}-i m_{I} \gamma_{5}\right) \psi=0
$$

can be rewritten in the form

$$
i \frac{\partial \psi}{\partial t}=\hat{H} \psi
$$

with $\hat{H}$ given by

$$
\hat{H}=\hat{\alpha} \cdot \hat{\mathbf{p}}+\hat{\beta} \hat{m}_{R}+i \hat{\gamma^{0}} \hat{\gamma^{5}} \hat{m}_{I}
$$

We now use the method of evenisation and evenise $\hat{H}^{2}$. We discuss later why we evenise $\hat{H}^{2}$ rather than $\hat{H}$.

We first write after some algebra,

$$
\hat{H}^{2}=\hat{\mathbf{p}}^{2}+|\hat{m}|^{2}+\hat{\alpha} \hat{\beta}\left[\hat{\mathbf{p}}, \hat{m}_{R}\right]+i \hat{\alpha} \hat{\gamma}^{0} \hat{\gamma}^{5}\left[\hat{\mathbf{p}}, \hat{m}_{I}\right]
$$

where, $|\hat{m}|^{2}=\hat{m}_{R}^{2}+\hat{m}_{I}^{2}$. Note that $\hat{\alpha}$ and $\hat{\mathbf{p}}$ are contracted in the last two terms on the right hand side. We take the wall to be planar in the $x-y$ plane and so $\hat{m}$ is a function of $\hat{z}$ only. Then the Hamiltonian can be written as

$$
\hat{H}^{2}=\hat{\mathbf{p}}^{2}+|\hat{m}|^{2}-\left(\hat{\alpha}^{3} \hat{\gamma}^{5}\right) \hat{\gamma}^{0} \hat{\gamma}^{5}\left(-i \hbar \partial_{z} \hat{m}_{R}\right)-i\left(\hat{\alpha^{3}} \hat{\gamma}^{5}\right) \hat{\beta}\left(-i \hbar \partial_{z} \hat{m}_{I}\right)
$$

where $z \equiv x^{3}$ and $\partial_{z} \equiv \partial / \partial \hat{x}^{3}$. Defining the spin operator $\hat{\mathbf{S}}$ as $\hat{\alpha} \hat{\gamma}^{5}$ (without the standard $\hbar / 2$ in the definition to be consistent with the notation in Refs. [2, 4]; the spin operator defined here is $\hat{\boldsymbol{\Sigma}}$ with Pauli matrices along the diagonal) the Hamiltonian can be rewritten as

$$
\hat{H}^{2}=\hat{\mathbf{p}}^{2}+|\hat{m}|^{2}-\hat{S}^{3} \hat{\gamma}^{0} \hat{\gamma}^{5}\left(-i \hbar \partial_{z} \hat{m}_{R}\right)-i \hat{S^{3}} \hat{\beta}\left(-i \hbar \partial_{z} \hat{m}_{I}\right)
$$

We chose to express $\hat{\alpha}^{3} \hat{\beta}$ and $\hat{\alpha}^{3} \hat{\gamma}^{0} \hat{\gamma}^{5}$ in terms of $\hat{S}^{3}$ as this simplifies the evenisation of the corresponding terms below.

We now evenise $\left[\hat{H}^{2}\right]$ to order $\hbar$. The spin operator in general does not commute with the Hamiltonian. In order to simplify the problem, as in Ref.[2], we assume that we are

\footnotetext{
${ }^{1}$ Alternate ways of writing the mass terms are $-|m| \bar{\psi} e^{i \theta \gamma_{5}} \psi$ and $-m \bar{\psi} P_{R} \psi-m^{*} \bar{\psi} P_{L} \psi$, where $m=|m| e^{i \theta}=$ $m_{R}+i m_{I}$, as in Refs. 2, 4].
} 
in an inertial reference frame where $x$ and $y$ components of the momentum are zero and consequently $\hat{\alpha}^{1}$ and $\hat{\alpha}^{2}$ will be absent from the Hamiltonian. This will allow us to set $\left\{S^{3}\right\}$ to 0 later. As mentioned earlier, we wish to express $\left[\hat{H}^{2}\right]$ in terms of $\left[\hat{p}_{z}\right]$ and $[\hat{z}]$ (where $p_{z} \equiv p^{3}$ ). The odd part of $\hat{p}_{z}$ will be proportional to $\left[\hat{\Lambda}, \hat{p}_{z}\right]$ (see Eq. (44) above) and so will be of order $\hbar$. Therefore, using Eq. (5), we can approximate $\left[\hat{p}_{z}^{2}\right]$ by $\left[\hat{p}_{z}\right]^{2}$. Similarly we approximate $\left[m_{R}^{2}(\hat{z})+m_{I}^{2}(\hat{z})\right]$ as $\left[m_{R}(\hat{z})\right]^{2}+\left[m_{I}(\hat{z})\right]^{2}$. Furthermore, since $\{\hat{z}\}$ is $O(\hbar)$, $\left[m_{R}(\hat{z})\right]=m_{R}([\hat{z}])+O\left(\hbar^{2}\right)$ as any dependence of the even operator $\left[m_{R}(\hat{z})\right]$ on the odd operator $\{\hat{z}\}$ can only appear as $\{\hat{z}\}^{2}$.

The last two terms on the right hand side of Eq. (12) are $O(\hbar)$ and therfore we will use the sign operator defined upto $O\left(\hbar^{0}\right)$ to evenise these terms. We define the zeroth order energy as

$$
E_{0}=\sqrt{p_{z}^{2}+|m|^{2}}
$$

where $p_{z}{ }^{2}$ and $|m|^{2}$ are real numbers and are expectation values of the corresponding operators in an eigenstate of definite energy and spin. (^designates an operator.) With this we define the sign operator upto $O\left(\hbar^{0}\right)$ as follows

$$
\hat{\Lambda}_{0}=\frac{\hat{H}}{\sqrt{p_{z}^{2}+|m|^{2}}}
$$

Note that $\left(\hat{\Lambda}_{0}\right)^{2}=1+O(\hbar)$ which we shall use in the derivation of evenised operators below.

Now

$$
\left[\hat{S}^{3}\right]=\left[\hat{\alpha}^{3} \hat{\gamma}^{5}\right]=\hat{S}^{3},
$$

i.e., $\left\{\hat{S}^{3}\right\}=0$ as $\hat{S}^{3}$ commutes with the Hamiltonian (which is true only in this preferred inertial frame), and

$$
\begin{gathered}
{\left[\hat{\gamma}^{0} \hat{\gamma}^{5}\right]=-i \frac{\hat{m}_{I}}{E_{0}} \hat{\Lambda}_{0}+O(\hbar)} \\
{[\hat{\beta}]=\frac{\hat{m}_{R}}{E_{0}} \hat{\Lambda}_{0}+O(\hbar) .}
\end{gathered}
$$

Derivations of the above expressions are provided in the Appendix. Thus

$$
\left[\hat{H}^{2}\right]=\left[\hat{p}_{z}\right]^{2}+\hat{m}_{R}^{2}+\hat{m}_{I}^{2}-\hbar \hat{S}^{3} \frac{\hat{m}_{I}}{E_{0}} \partial_{z} \hat{m}_{R} \hat{\Lambda}_{0}-\hbar \hat{S}^{3} \frac{\hat{m}_{R}}{E_{0}} \partial_{z} \hat{m}_{I} \hat{\Lambda}_{0}
$$

where all mass functions are functions of $[\hat{z}]$. Replacing evenised operators $[\hat{z}]$ and $\left[\hat{p}_{z}\right]$ by real numbers representing their expectation values in states of definite energy and spin, 
$|E, s\rangle$ for particles and $|-E,-s\rangle$ for antiparticles, we deduce the corresponding expression for the energy to be

$$
E^{2}=p_{z}^{2}+|m|^{2}-\frac{\hbar s}{E_{0}}\left(m_{I} \partial_{z} m_{R}-m_{R} \partial_{z} m_{I}\right)
$$

where we have replaced $\hat{S}^{3}$ by its eigenvalues $\pm s$ and $\Lambda_{0}$ by \pm 1 for particle/antiparticle states. Writing $m_{R}=|m| \cos \theta$ and $m_{I}=|m| \sin \theta$ one can rewrite the above equation as

$$
E^{2}=p_{z}^{2}+|m|^{2}-\frac{\hbar s}{E_{0}}|m|^{2} \theta^{\prime}
$$

The energy relation for both particles and antiparticles can then be written upto order $\hbar$ as

$$
E=\sqrt{p_{z}^{2}+|m|^{2}}-\frac{\hbar s}{2 E_{0}^{2}}|m|^{2} \theta^{\prime}
$$

This expression for the energy in terms of the position, momentum and spin of the particles/antiparticles is the same as the energy relation obtained in Ref. [3]. Note that if we had wished to obtain the energy relation to $O(\hbar)$ by evenising $\hat{H}$ instead of evenizing $\hat{H}^{2}$ we would have faced a problem as we would have needed $\hat{\Lambda}$ to $O(\hbar)$ which itself requires $E$ to $O(\hbar)$. However, since $\{\hat{H}\}=0,[\hat{H}]=\left[\hat{H}^{2}\right]^{1 / 2}$. We are now able to define the sign operator upto $O(\hbar)$ as $\hat{\Lambda}=\hat{H} / E$ and use the same to obtain the evenised velocity and force to $O(\hbar)$ for the kinetic equation.

We now obtain the kinetic equation. Using the chain rule for partial differentiation the kinetic equation, in the absence of collisions, is written as

$$
\partial_{t} f_{s \pm}+\frac{d z}{d t} \partial_{z} f_{s \pm}+\frac{d p_{z}}{d t} \partial_{p_{z}} f_{s \pm}=0
$$

where $f_{s \pm}$ are the particle and antiparticle distribution functions. We will associate $d z / d t$ and $d p_{z} / d t$ with the expectation values of $[d \hat{z} / d t]$ and $\left[d \hat{p}_{z} / d t\right]$ in states of definite energy and spin as before. Implicitly we are assuming here that the form of the quantum Boltzmann equation is the same as that of the classical Boltzmann equation and the quantum corrections are contained only in the coefficients of the equation, namely, in the expression for the velocity and the force. The evenised expressions for the velocity and force to $O(\hbar)$ are derived in the Appendix as

$$
\begin{aligned}
{[d \hat{z} / d t] } & =\left[-\frac{i}{\hbar}[\hat{z}, \hat{H}]\right] \\
& =\frac{\left[\hat{p}_{z}\right]}{E} \hat{\Lambda}
\end{aligned}
$$




$$
\begin{aligned}
{\left[d \hat{p}_{z} / d t\right] } & =\left[-\frac{i}{\hbar}\left[\hat{p}_{z}, \hat{H}\right]\right] \\
& =\left(-\frac{|\hat{m}|^{2^{\prime}}}{2 E}+\frac{\hbar \hat{S}^{3} \hat{\Lambda}_{0}\left(|\hat{m}|^{2} \hat{\theta}^{\prime}\right)^{\prime}}{2 E^{2}}\right) \hat{\Lambda}
\end{aligned}
$$

where $|\hat{m}|$ and $\hat{\theta}^{\prime}$ are functions of $[\hat{z}]$ and $E$ is as given in Eq. (21). Substituting the eigenvalues of the above operators in the kinetic equation ${ }^{2}$, we get

$$
\partial_{t} f_{s \pm}+\frac{p_{z}}{E} \partial_{z} f_{s \pm}+\left(-\frac{|m|^{2^{\prime}}}{2 E}+\frac{\hbar s\left(|m|^{2} \theta^{\prime}\right)^{\prime}}{2 E^{2}}\right) \partial_{p_{z}} f_{s \pm}=0
$$

We note that this agrees with the kinetic equation obtained in Ref. [3]. ${ }^{3}$

Several comments are in order here. If we apply Hamilton's equations to the energy relation in Eq. (21), i.e., $d z / d t=\partial E / \partial p_{z}$ and $d p_{z} / d t=-\partial E / \partial z$, we get expressions for $d z / d t$ and $d p_{z} / d t$ that differ from the expectation values of the corresponding evenised operators $[d \hat{z} / d t]$ and $\left[d \hat{p}_{z} / d t\right]$. There can be two reasons for this. Firstly, the real number $p_{z}$ appearing above in Eq. (21) is not the canonical momentum. It represents the expectation value of $\left[\hat{p}_{z}\right]$ whereas the canonical momentum would be associated with the expectation value of $\hat{p}_{z}$. Secondly, ignoring for now the distinction between $\hat{p}_{z}$ and $\left[\hat{p}_{z}\right]$, Ehrenfest's theorem for a relativistic system would imply $d\langle\hat{z}\rangle / d t=\left\langle\partial \hat{H} / \partial \hat{p}_{z}\right\rangle$ and $d\left\langle\hat{p}_{z}\right\rangle / d t=-\langle\partial \hat{H} / \partial \hat{z}\rangle$, and not $d\langle\hat{z}\rangle / d t=\partial\langle\hat{H}\rangle / \partial\left\langle\hat{p}_{z}\right\rangle$ and $d\left\langle\hat{p}_{z}\right\rangle / d t=-\partial\langle\hat{H}\rangle / \partial\langle\hat{z}\rangle$.

In the literature 2, 4] there has been discussion on identifying the canonical and kinetic momentum of the particle. One may presume that since $\hat{p}_{z}$ is identified with the $-i \hat{\partial}_{z}$ operator it is conjugate to $\hat{z}$ and represents the canonical momentum. Now our energy relation agrees with that of Ref. [3]. But the energy relation in Ref. 3] is necessarily in terms of the kinetic momentum as the Wigner function invoked in Ref. 3] is always written in terms of the kinetic momentum of the system (see Sec. 3 of Ref. [13]). This indicates that our real number $p_{z}=\left\langle\left[\hat{p}_{z}\right]\right\rangle$ in the energy relation above is the kinetic momentum of Ref. [3]. It is not obvious to us why the evenised canonical momentum in this problem becomes equivalent to the kinetic momentum.

\footnotetext{
2 The overall minus sign in the expectation value for the velocity and force for antiparticles can be absorbed by taking the expectation value of the momentum in antiparticle states to be $-p_{z}$, as for systems with constant mass, where the energy eignenstates are also momentum eigenstates.

${ }^{3}$ In the earlier version of this paper, we had identified antiparticles of spin $s$ with negative energy particle solutions of spin $s$, rather than $-s$ (see Sec. 7.1 of Ref. [1]). Hence the expressions for energy and force were different for particles and antiparticles of the same spin. Our earlier results agreed with Refs. 2, 4] which perhaps contain the same misinterpretation. Our current results reflect the $P$ and $C P$ violation and $C$ conservation properties of the Lagrangian in Eq. (6).
} 
If one wishes to define a classical Hamiltonian function which gives the expressions for velocity and force above by applying Hamilton's equations, one may define a canonical momentum $p_{c}$ by the following ansatz of Eq. (61) of Ref. [14]

$$
p_{c}=p_{z}\left(1+\frac{\hbar s \theta^{\prime}}{2\left(p_{z}^{2}+|m|^{2}\right)^{\frac{1}{2}}}\right)
$$

and reexpress the energy relation in terms of $p_{c}$ as

$$
\begin{aligned}
E & =\sqrt{p_{z}^{2}\left(1+\frac{\hbar s \theta^{\prime}}{E_{0}}\right)+|m|^{2}}-\frac{\hbar s \theta^{\prime}}{2} \\
& =\sqrt{p_{c}^{2}+|m|^{2}}-\frac{\hbar s \theta^{\prime}}{2} .
\end{aligned}
$$

Then $d z / d t=\partial E / \partial p_{c}$ and, rewriting Eq. (26) as $p_{z}=\mathrm{Fac} p_{c}, d p_{z} / d t=(d \mathrm{Fac} / d t) * p_{c}-\mathrm{Fac} *$ $\partial E / \partial z$. These agree with the expressions of the evenised velocity and force above. Eq. (26) agrees with the relation between kinetic and canonical momentum in Ref. [4], with $\alpha_{C P}$ of Ref. [4] set to 0 .

\section{CONCLUSION}

In conclusion, we have provided an alternate procedure for obtaining the energy relation and the kinetic equation for fermions and antifermions interacting with the Higgs bubble wall during the electroweak phase transition using the method of evenisation. Our derivation of the single particle behaviour using the Heisenberg equations of motion has allowed us to include quantum corrections systematically to $O(\hbar)$. This provides a direct and intuitive way of obtaining the kinetic equation in this limit. Our results agree with those that may be

obtained using more formal quantum field theoretic methods involving the Wigner function, or the WKB method. In particular, we rederive the semi-classical force that may be obtained by these other methods.

\section{Acknowledgments}

We would like to thank Tomislav Prokopec and Steffen Weinstock for many clarifications about their work and for many useful comments regarding an earlier version of this work. We would also like to thank B. Ananthanarayan for fruitful discussions. R.R. would like to thank N. P. M. Nair for help with preparation of the manuscript. 


\section{Appendix}

We present below the derivations of the various evenised expressions quoted in the text.

Standard formulae that we shall use are $\hat{\beta}^{2}=-\left(\hat{\gamma}^{0} \hat{\gamma}^{5}\right)^{2}=\left(\hat{\alpha}^{3}\right)^{2}=1$. The symbols square brackets are used below for both commutators and evenised operators but the difference is clear from the context.

Evenised $\hat{\beta}$ and $\hat{\gamma}^{0} \hat{\gamma}^{5}$ to $O\left(\hbar^{0}\right)$ :

$$
\begin{aligned}
2[\hat{\beta}] & =\hat{\beta}+\hat{\Lambda}_{0} \hat{\beta} \hat{\Lambda}_{0} \\
& =\hat{\beta}+\frac{\hat{\alpha}^{3} p^{3}+\hat{\beta} \hat{m}_{R}+i \hat{\gamma}^{0} \hat{\gamma}^{5} \hat{m}_{I}}{E_{0}} \hat{\beta} \hat{\Lambda}_{0} \\
& =\hat{\beta}+\hat{\beta} \frac{-\hat{\alpha}^{3} p^{3}+\hat{\beta} \hat{m}_{R}-i \hat{\gamma}^{0} \hat{\gamma}^{5} \hat{m}_{I}}{E_{0}} \hat{\Lambda}_{0} \\
& =\hat{\beta}+\hat{\beta} \frac{-\hat{\Lambda}_{0} E_{0}+2 \hat{\beta} \hat{m}_{R}}{E_{0}} \hat{\Lambda}_{0} \\
& =\frac{2 \hat{m}_{R}}{E_{0}} \hat{\Lambda}_{0},
\end{aligned}
$$

where we have used $\hat{\Lambda}_{0}^{2}=1+O(\hbar)$ in the last equality. Since the lhs is even and the sign operator on the rhs is even, $m_{R}(\hat{z})$ may be replaced by $\left[m_{R}(\hat{z})\right]$, which, as explained in the text, is equal to $m_{R}([\hat{z}])$ to $O(\hbar)$. Therefore

$$
[\hat{\beta}]=\frac{m_{R}([\hat{z}])}{E_{0}} \hat{\Lambda}_{0}+O(\hbar) .
$$

Similarly

$$
\begin{aligned}
2\left[\hat{\gamma}^{0} \hat{\gamma}^{5}\right] & =\hat{\gamma}^{0} \hat{\gamma}^{5}+\hat{\Lambda}_{0} \hat{\gamma}^{0} \hat{\gamma}^{5} \hat{\Lambda}_{0} \\
& =\hat{\gamma}^{0} \hat{\gamma}^{5}+\hat{\gamma}^{0} \hat{\gamma}^{5} \frac{-\hat{\Lambda}_{0} E_{0}+2 \hat{\gamma}^{0} \hat{\gamma}^{5} i \hat{m}_{I}}{E_{0}} \hat{\Lambda}_{0} \\
& =-\frac{2 i \hat{m}_{I}}{E_{0}} \hat{\Lambda}_{0} .
\end{aligned}
$$

Therefore, replacing $m_{I}(\hat{z})$ by $m_{I}([\hat{z}])$,

$$
\left[\hat{\gamma}^{0} \hat{\gamma}^{5}\right]=-\frac{i m_{I}([\hat{z}])}{E_{0}} \hat{\Lambda}_{0}+O(\hbar) .
$$

Evenised expressions for the velocity and the force to $O(\hbar)$ :

Since we wish to work till $O(\hbar)$ we now use the sign operator $\hat{\Lambda}$ defined as

$$
\hat{\Lambda}=\frac{\hat{H}}{E}
$$


with $E$ defined to $O(\hbar)$ as in Eq. (21). Now $d \hat{z} / d t=-(i / \hbar)[\hat{z}, \hat{H}]=\hat{\alpha}^{3}$. Then

$$
\begin{aligned}
2\left[\hat{\alpha}^{3}\right] & =\hat{\alpha}^{3}+\hat{\Lambda} \hat{\alpha}^{3} \hat{\Lambda} \\
& =\hat{\alpha}^{3}+\hat{\alpha}^{3} \frac{-\hat{\Lambda} E+2 \hat{\alpha}^{3} \hat{p}^{3}}{E} \Lambda \\
& =\frac{2 \hat{p}^{3}}{E} \hat{\Lambda}=\frac{2 \hat{p}_{z}}{E} \hat{\Lambda} .
\end{aligned}
$$

Since the lhs of the above equation and the sign operator on the rhs are even we may replace $\hat{p}_{z}$ by $\left[\hat{p}_{z}\right]$. Therefore we get

$$
[d \hat{z} / d t]=\frac{\left[\hat{p}_{z}\right]}{E} \hat{\Lambda}
$$

The force $d \hat{p}_{z} / d t=-(i / \hbar)\left[\hat{p}_{z}, \hat{H}\right]=-\hat{\beta} \hat{m}_{R}^{\prime}-i \hat{\gamma}^{0} \hat{\gamma}^{5} \hat{m}_{I}^{\prime}$. Then

$$
\begin{aligned}
2\left[\hat{\beta} \hat{m}_{R}^{\prime}\right] & =\hat{\beta} \hat{m}_{R}^{\prime}+\hat{\Lambda} \hat{\beta} \hat{m}_{R}^{\prime} \hat{\Lambda} \\
& =\hat{\beta} \hat{m}_{R}^{\prime}+\hat{\beta} \hat{m}_{R}^{\prime} \frac{-\hat{\Lambda} E+2 \hat{\beta} \hat{m}_{R}}{E} \hat{\Lambda}-\hat{\beta} \hat{\alpha}^{3} \frac{\left[\hat{p}_{z}, \hat{m}_{R}^{\prime}\right]}{E} \hat{\Lambda} \\
& =\frac{2 \hat{m}_{R}^{\prime} \hat{m}_{R}+i \hbar \hat{\beta} \hat{\alpha}^{3} \hat{m}_{R}^{\prime \prime} \hat{\Lambda} .}{E} .
\end{aligned}
$$

Again, we may replace $\hat{m}_{R}^{\prime} \hat{m}_{R}$ by $\left[\hat{m}_{R}^{\prime} \hat{m}_{R}\right]$ as both sides of the equation should be even. As $\left\{\hat{m}_{R}^{\prime}\right\}\left\{\hat{m}_{R}\right\}$ is $O\left(\hbar^{2}\right)$, Eq. (15) implies that this reduces to $\left[m_{R}^{\prime}(\hat{z})\right]\left[m_{R}(\hat{z})\right]$. This may further be rewritten as $m_{R}^{\prime}([\hat{z}]) m_{R}([\hat{z}])$, since as discussed earlier in the text, the dependence on $\{z\}$ of each term in the product will be at $O\left(\hbar^{2}\right)$. For the second term in the numerator we replace $\hat{\beta} \hat{\alpha}^{3} \hat{m}_{R}^{\prime \prime}$ by $\left[\hat{\beta} \hat{\alpha}^{3} \hat{m}_{R}^{\prime \prime}\right]$. Since this term is already of $O(\hbar)$ from the commutation employed in its derivation, we can then write the product as $\left[\hat{\beta} \hat{\alpha}^{3}\right]\left[m_{R}^{\prime \prime}(\hat{z})\right]=\left[\hat{\beta} \hat{\alpha}^{3}\right] m_{R}^{\prime \prime}([\hat{z}])$. Now $\hat{\beta} \hat{\alpha}^{3}=\hat{S}^{3} \hat{\gamma}^{0} \hat{\gamma}^{5}$ and since $\left\{\hat{S}^{3}\right\}=0,\left[\hat{\beta} \hat{\alpha}^{3}\right]=\hat{S}^{3}\left[\hat{\gamma}^{0} \hat{\gamma}^{5}\right]=-i \hat{S}^{3} \frac{m_{I}([\hat{z}])}{E_{0}} \hat{\Lambda}_{0}$. Therefore

$$
\left[\hat{\beta} \hat{m}_{R}^{\prime}\right]=\left(\frac{m_{R}^{2 \prime}([\hat{z}])}{2 E}+\frac{\hbar \hat{S}^{3} \hat{\Lambda}_{0} m_{I}([\hat{z}]) m_{R}^{\prime \prime}([\hat{z}])}{2 E^{2}}\right) \hat{\Lambda} .
$$

For the second term in $d \hat{p}_{z} / d t$,

$$
\begin{aligned}
2\left[\hat{\gamma}^{0} \hat{\gamma}^{5} \hat{m}_{I}^{\prime}\right] & =\hat{\gamma}^{0} \hat{\gamma}^{5} \hat{m}_{I}^{\prime}+\hat{\Lambda} \hat{\gamma}^{0} \hat{\gamma}^{5} \hat{m}_{I}^{\prime} \hat{\Lambda} \\
& =\hat{\gamma}^{0} \hat{\gamma}^{5} \hat{m}_{I}^{\prime}+\hat{\gamma}^{0} \hat{\gamma}^{5} \hat{m}_{I}^{\prime} \frac{-\hat{\Lambda} E+2 i \hat{\gamma}^{0} \hat{\gamma}^{5} \hat{m}_{I}}{E} \hat{\Lambda}-\hat{\gamma}^{0} \hat{\gamma}^{5} \hat{\alpha}^{3} \frac{\left[\hat{p}_{z}, \hat{m}_{I}^{\prime}\right]}{E} \Lambda \\
& =\left(\frac{-2 i \hat{m}_{I}^{\prime} \hat{m}_{I}+i \hbar \hat{\gamma}^{0} \hat{\gamma}^{5} \hat{\alpha}^{3} \hat{m}_{I}^{\prime \prime} \hat{\Lambda} .}{E}\right.
\end{aligned}
$$

$\hat{\gamma}^{0} \hat{\gamma}^{5} \hat{\alpha}^{3}=\hat{S}^{3} \hat{\beta}$. Invoking arguments as above and substituting for $[\hat{\beta}]$ we get

$$
\left[i \hat{\gamma}^{0} \hat{\gamma}^{5} \hat{m}_{I}^{\prime}\right]=\left(\frac{m_{I}^{2 \prime}([\hat{z}])}{2 E}-\frac{\hbar \hat{S}^{3} \hat{\Lambda}_{0} m_{R}([\hat{z}]) m_{I}^{\prime \prime}([\hat{z}])}{2 E^{2}}\right) \hat{\Lambda}
$$


Thus

$$
\left[d \hat{p}_{z} / d t\right]=\left(-\frac{|\hat{m}|^{2^{\prime}}}{2 E}+\frac{\hbar \hat{S}^{3} \hat{\Lambda}_{0}\left(|\hat{m}|^{2} \hat{\theta}^{\prime}\right)^{\prime}}{2 E^{2}}\right) \hat{\Lambda}
$$

where we have used $\hat{m}_{R} \hat{m}_{I}^{\prime \prime}-\hat{m}_{I} \hat{m}_{R}^{\prime \prime}=\left(|\hat{m}|^{2} \hat{\theta}^{\prime}\right)^{\prime}$. $|\hat{m}|$ and $\hat{\theta}^{\prime}$ above are functions of $[\hat{z}]$.

[1] W. Greiner, Relativistic Quantum Mechanics, Springer-Verlag, Berlin, Third Edition (2000) (Ch. 2).

[2] K. Kainulainen, T. Prokopec, M. G. Schmidt and S. Weinstock, JHEP 0106 (2001) 31 arXiv:hep-ph/0105295.

[3] T. Prokopec, M. G. Schmidt and S. Weinstock, Ann. Phys. 314 (2004) 208 arXiv:hep-ph/0312110.

[4] J. M. Cline, M. Joyce and K. Kainulainen, JHEP 0007 (2000) 018 arXiv:hep-ph/0006119; erratum, arXiv:hep-ph/0110031.

[5] M. Joyce, T. Prokopec and N. Turok, Phys. Rev. D 53 (1996) 2958 arXiv:hep-ph/9410282.

[6] J. M. Cline, M. Joyce and K. Kainulainen, Phys. Lett. B417 (1998) 79 arXiv:hep-ph/9708393; erratum, Phys. Lett. B448 (1999) 321.

[7] P. Huet and A. E. Nelson, Phys. Rev. D 53 (1996) 4578 arXiv:hep-ph/9506477.

[8] A. Riotto, Phys. Rev. D 53 (1996) 5834 arXiv:hep-ph/9510271.

[9] A. Riotto, Nucl. Phys. B518 (1998) 339 arXiv:hep-ph/9712221.

[10] A. Riotto, Phys. Rev. D 58 (1998) 095009 arXiv:hep-ph/9803357.

[11] M. Carena, J.M. Moreno, M. Quiros, M. Seco and C.E.M. Wagner, Nucl. Phys. B599 (2001) 158 arXiv:hep-ph/0011055.

[12] M. Joyce, T. Prokopec and N. Turok, Phys. Rev. Lett. 75 (1995) 1695 arXiv:hep-ph/9408339.

[13] D. Vasak, M. Gyulassy and H.-T. Elze, Annals of Physics 173 (1987) 462.

[14] K. Kainulainen, T. Prokopec, M. G. Schmidt and S. Weinstock, Phys. Rev. D 66 (2002) 043502 arXiv:hep-ph/0202177. 